\title{
Physical Fidelity in Particle-In-Cell Modeling of Small Debye-Length Plasmas
}

\author{
B. A. Shadwick* and C. B. Schroeder ${ }^{\dagger}$ \\ ${ }^{*}$ Department of Physics and Astronomy, University of Nebraska, Lincoln, Nebraska 68588-0111 \\ ${ }^{\dagger}$ Lawrence Berkeley National Laboratory, Berkeley, California 94720
}

\begin{abstract}
The connection between macro-particle shape functions and non-physical phase-space "heating" in the particle-in-cell (PIC) algorithm is examined. The development of fine-scale phasespace structures starting from a cold initial condition is shown to be related to spatial correlations in the interpolated fields used in the Lorentz force. It is shown that the plasma evolution via the PIC algorithm from a cold initial condition leads to a state that is not consistent with that of a thermal plasma.
\end{abstract}

PACS: 52.38.-r, 52.65.Rr

\section{INTRODUCTION}

Particle-in-cell (PIC) codes are commonly used tools to study kinetic effects in plasmas, that is, behavior beyond that described by a moment-based description of the phasespace distribution. The PIC [1] method, originally developed for hydrodynamics by Harlow [2] was introduced to plasma physics in the early days of the controlled fusion program and has been widely applied to the study of fusion plasmas, astrophysical plasma, plasma processing, and beam devices [3, 4]. In broad terms, fusion plasmas are hot (ion temperatures in the multi- $\mathrm{kV}$ range) and the plasma flow is typically turbulent. As the accelerator applications of short-pulse laser-plasma interactions developed, it was natural to apply this well-known tool to these novel systems. In short-pulse laser-plasma interactions, the plasma is collisionless on the time-scales of interest (e.g., the laser duration), and the plasmas are typically photo-ionized, with initial temperatures on the order of the ionization potential, i.e., tens of eV. Unlike tokamak plasmas, the plasma response to a short laser pulse can be quite laminar [5,6], and there is no heating.

When studying short-pulse laser-plasma interactions, PIC simulations are commonly initialized cold. With a cold initial condition, as we will see below, the "heating" in PIC is the result of algorithmic artifacts; a proper thermal state is not generated and the plasma response is not correct. Under these circumstances, the trapping of particles is not due to the capture of the tails of a thermal distribution (as in a real plasma) $[7,8]$, but to the capture of particles from the bulk of the distributions that are on incorrect phase-space orbits. In one-dimension (1-D) it has been shown that the PIC model can reproduce the response of a thermal plasma to a short laser pulse [6]. 


\section{NUMERICAL HEATING MECHANISMS}

Unavoidable discretization of the physical model and the necessarily small number of macro-particles relative to the number of physical electrons both give rise to unphysical heating $[1,9]$. Figure 1 shows the evolution in RMS momentum spread of a thermal plasma without any drive versus time and illustrates two numerical heating mechanisms: scattering and grid heating. While both mechanisms illustrated in Fig. 1 are unphysical, they have distinct origins. Scattering is a "discrete particle effect" related to approximating the continuous phase-space density by individual macro-particles. As macroparticles drift from one cell to another in simulation of an unforced thermal plasma, there will be fluctuations in the number of macro-particles per cell. Since the average number of macro particles per cell is rather small, even fluctuations of \pm 1 macro-particle can lead to a large potential. These large, approximately random, fluctuations in potential lead to a localized electric field which acts on the macro-particles. In effect, the macro-particles scatter off fluctuations in the potential. Grid heating is a kinetic instability resulting from the grid aliasing high-frequency modes (not resolved by the grid) to low frequencies $[1,10]$. The modification of the usual warm-plasma dispersion relation by aliasing effects results in roots with positive imaginary parts. Since this numerical instability arises from the same basic physical processes (but triggered by grid-aliasing) as true plasma modes, it can both mimic real plasma phenomena and act to drive other, physical plasma responses. The growth rate of this instability is significantly reduced once the plasma temperature reaches a level given by $\lambda_{D} k_{g} \approx 1$, where $k_{g}=\pi / \Delta x$ is the smallest wavenumber of the supported by the grid and $\lambda_{D}$ is the plasma Debye length. Common to both mechanism is the creation of particle energy; likewise both mechanisms results in dynamical evolution of a state that physically is an equilibrium, but, evidently, not a fixed-point of the PIC algorithm. Furthermore, both mechanisms represent linear instabilities (in the von Neumann sense [11]) of the numerical method.

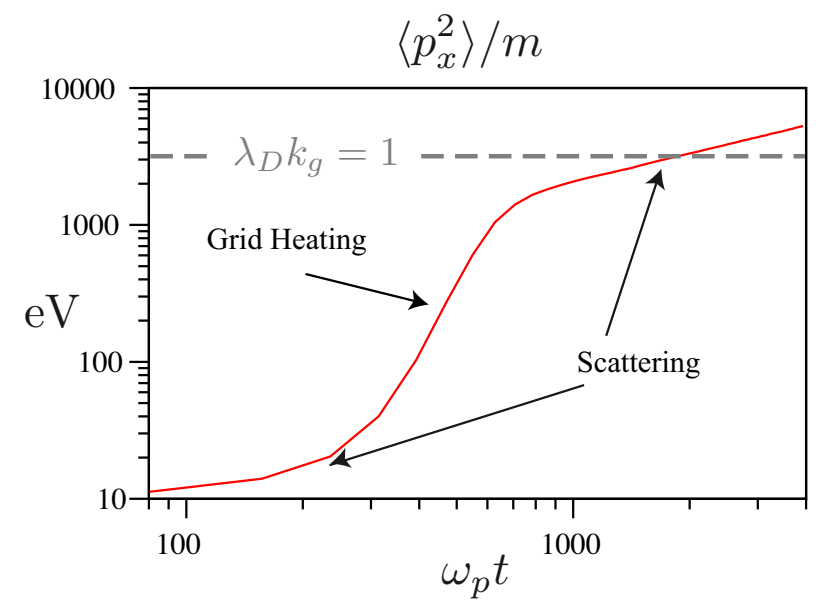

FIGURE 1. An illustration of numerical heating mechanisms in PIC simulations. An initially thermal plasma is allowed to evolve without excitation. The total kinetic energy of the system exhibits a secular growth in time. As indicated, the regions of differing heating rates correspond to distinct heating mechanisms. 

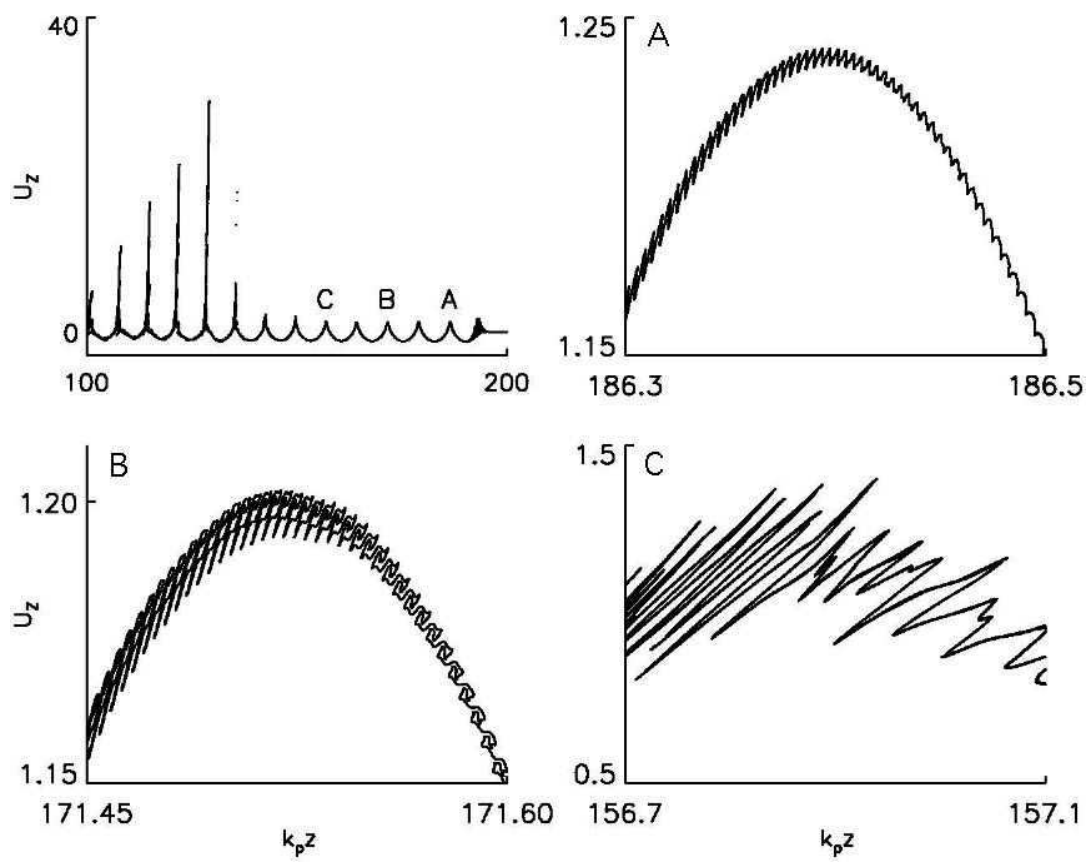

FIGURE 2. Macro-particle phase space of a laser-driven plasma wave in 1-D for a plasma initially at rest. The longitudinal grid spacing in the simulation was $\lambda_{0} / 36$ with 400 macro-particles per cell. The boxes $\mathrm{A}, \mathrm{B}$, and $\mathrm{C}$ show detailed of phase space at the first, third, and fifth bucket after the laser pulse. For these initial conditions, no physical trapping should occur and the plasma response should be purely hydrodynamic. See Fig. 5 of Ref. [12].

These numerical heating mechanisms will alter phase space and can mimic physical processes leading to incorrect interpretation of computational results [12]. This will be of particular importance when attempting to model detailed kinetic effects, such as trapping of the plasma electrons and generation of dark current in a plasma accelerator [7,8]. In addition, since the macro-particle positions are not restricted to mesh points, some form of interpolation is necessary to evaluate the force, resulting in trajectory errors.

Figure 2 shows the longitudinal phase space of macro-particles in a 1-D PIC simulation of a laser-plasma interaction with $\omega_{0} / \omega_{p}=10, a_{0}=2$, and $k_{p} L=2$. The simulation used a longitudinal grid spacing of $\lambda_{0} / 36$ and 400 macro-particles per cell. What begins as high-frequency oscillations [Fig. 2(A)] in the plasma momentum develop into a structure on phase space that corresponds to a multi-peak distribution function resembling Dawson's cold beams [13]. The momentum spread that develops has been shown to be inconsistent [12] with the known thermal response of a Vlasov plasma to an intense, short laser pulse [5]. Thus this plasma state, which is the product of artifacts of the PIC algorithm, cannot be considered a "thermal plasma"; in fact, to refer to the processes leading to this phase space, starting from a cold initial condition, as heating is technically incorrect. In particular, there is no thermal initial condition (i.e., no isotropic plasma equilibrium) that will lead to the observed evolution.

Additionally, this evolution violates Gardner's restacking theorem [14], providing further evidence that the observed dynamics are inconsistent with the Vlasov-Maxwell equations. The dynamics illustrated in Fig. 2 will not, in general, lead to constant 


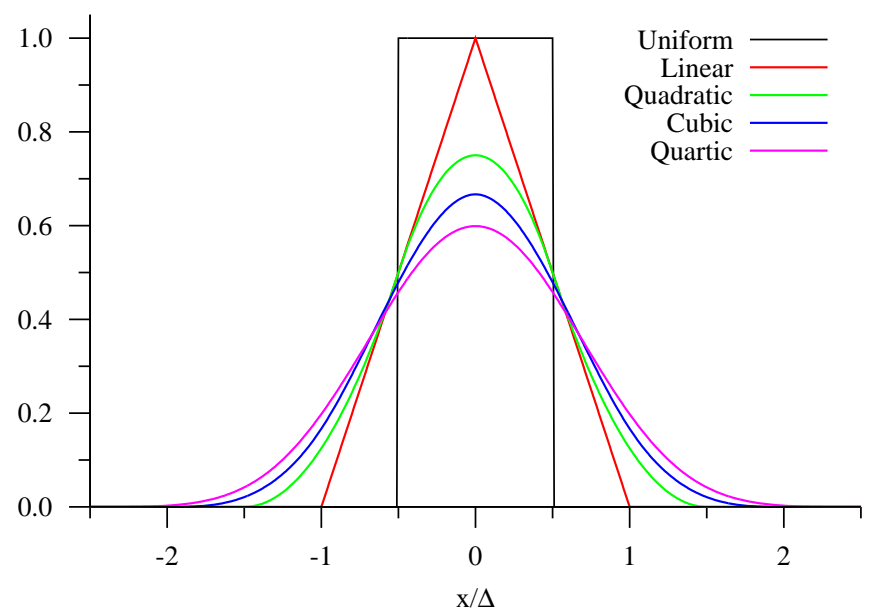

FIGURE 3. Particle shapes functions for various interpolation schemes. The higher order shape functions can be systematically obtained from the uniform shape (so called "nearest-grid-point" weighting) [9]. The horizontal axis is scaled relative to the grid spacing; smoother particle shapes result in larger macro-particles.
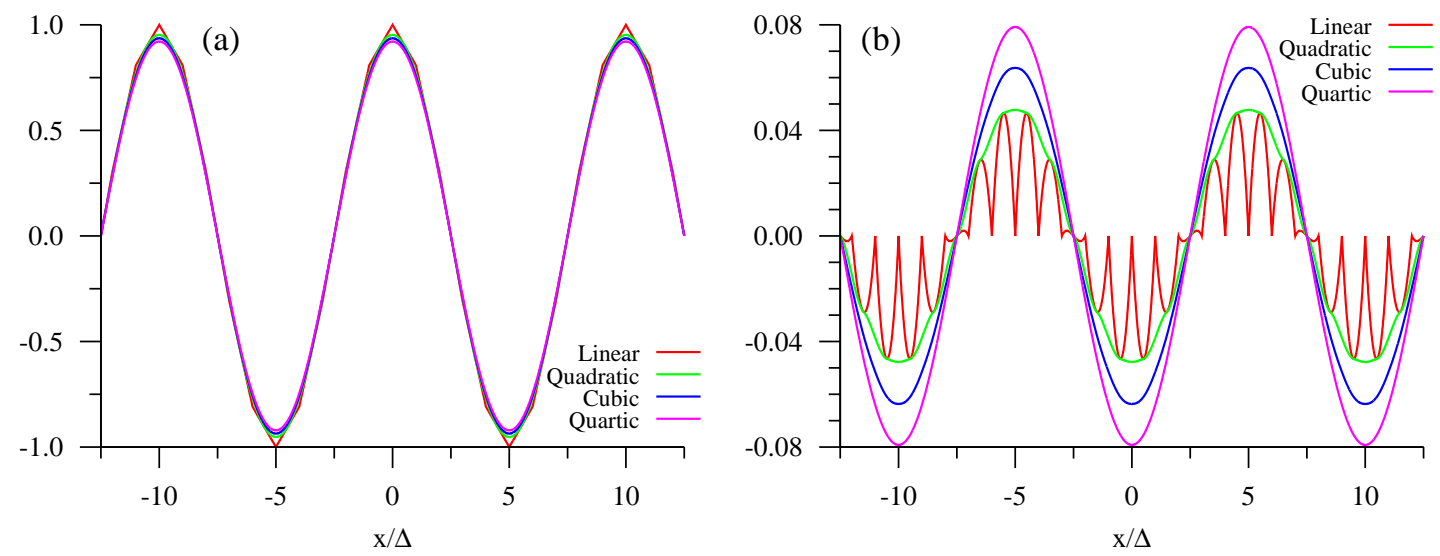

FIGURE 4. (a) Interpolation of $\cos (\pi x / 5 \Delta)$ using the particle shapes shown in Fig. 3. The smoothing effect of higher-order methods is clearly visible as is the fact the peak amplitude for these methods decreases with order. (b) Errors for the interpolation shown in (a). The higher-order methods systematically result in a great error at the maxima and minima of the interpolated function. The higher-order methods also lack the high frequency error components present in the linearly interpolated field.

values for the Casimirs of the Vlasov-Maxwell equations. Of these Casimirs, next to total particle number (which is conserved here), entropy has the most concrete physical interpretation. These dynamics, by allowing the distribution function to develop multiple peaks in momentum at a given spatial point, leads to large changes in the total entropy of the system. (The entropy in a collisionless plasma in constant.) Thus, we are lead to the conclusion that there is no initial condition (thermal or otherwise) that will lead to the observed dynamics.

To understand the origin of the structures in Fig. 2, it is instructive to examine the force interpolation mechanism in the PIC algorithm. Since the macro-particles are allowed to 
free move in phase space while the fields are only known on a spatial grid, it is necessary to provide field values at locations other than grid points and to determine the current at the grid points. The most elementary approach is the so-called nearest-grid-point (NGP) method where the fields at the nearest grid point to the macro-particle are used in the Lorentz force (and, likewise, the entire current associated with a given macro-particle is assigned to the nearest grid point). The large noise inherent in the NGP method lead to the development of "high-order" interpolation and current deposition $[9,15,16]$. The current is assigned to the spatial grid by the requirement of zero self-force. The result is that the interpolated fields are not, in general, consistent with the intra-cell current density. This zero self-force requirement appears to eliminate the use of derivative information to improve the accuracy of the interpolated fields (which would bring them closer to consistency with the intra-cell current density), as it is unclear whether it is possible to construct the required particle shape needed to accumulate the current.

Figure 3 shows the standard particle shape functions through quartic order. Figures 4(a) and 4(b), respectively, show the interpolation of a sinusoidal field field using these particle shapes and the resulting errors. The higher-order shape functions lead to smoother interpolation but not to higher accuracy; except for NGP, all the higher-order shapes lead to interpolations that are second-order accurate in the grid size. As can be seen in Fig. 4(b), as the order of the shape function grows, the errors become systematically larger (the coefficient in the error terms grows but the error remains second-order). One consequence is that the laser ponderomotive force is be systematically underestimated. Moreover, only linear interpolation results in zero error on the grid-points and as such, the error in the linear case contains frequencies larger than $k_{g}$.

The phase-space structures shown in Fig. 2 are not entirely the result of the heating mechanisms illustrated in Fig. 1. The initial evolution of the structure is solely due to spatial correlations in the interpolation error. Only once these orbit displacements grow to the point that, after having been "coarse-grained" to the grid, the resulting current distribution corresponds to a broadened phase-space distribution, does the collective instability responsible for grid heating grow. The fact that current smoothing is significantly less effective at suppressing the growth of these phase-space structures [12] suggests that this effect is due not to high-frequencies introduced in depositing current on the grid but rather to interpolation of the fields onto the particle locations (which is not affected by smoothing the current).

As shown in Fig. 5, these phase-space structures grow more slowly when higher-order interpolation is used. This is not the result of increased accuracy but smaller variation in the interpolation error across the cell. As the resolution is increased, for a given interpolation method, the absolute magnitude of the variation in interpolation error across the cell is reduced, leading to neighboring orbits having smaller relative displacements, slowing the growth of the structures. The ultimate cause of this phenomena is the need to approximate the fields between the grid points. By forcing greater continuity (at the cost of absolute accuracy), higher-order interpolation methods result in an intra-cell approximation for the fields that varies much less over the cell than for the linear case. Linear interpolation, which forces zero interpolation error at the grid points, leads to large relative errors and results in nearby macro-particles experiencing significantly different fields. Since these errors are correlated to the position in the cell, subsequent time steps tend to reinforce the error. Higher-order interpolation results in greater quantitative 

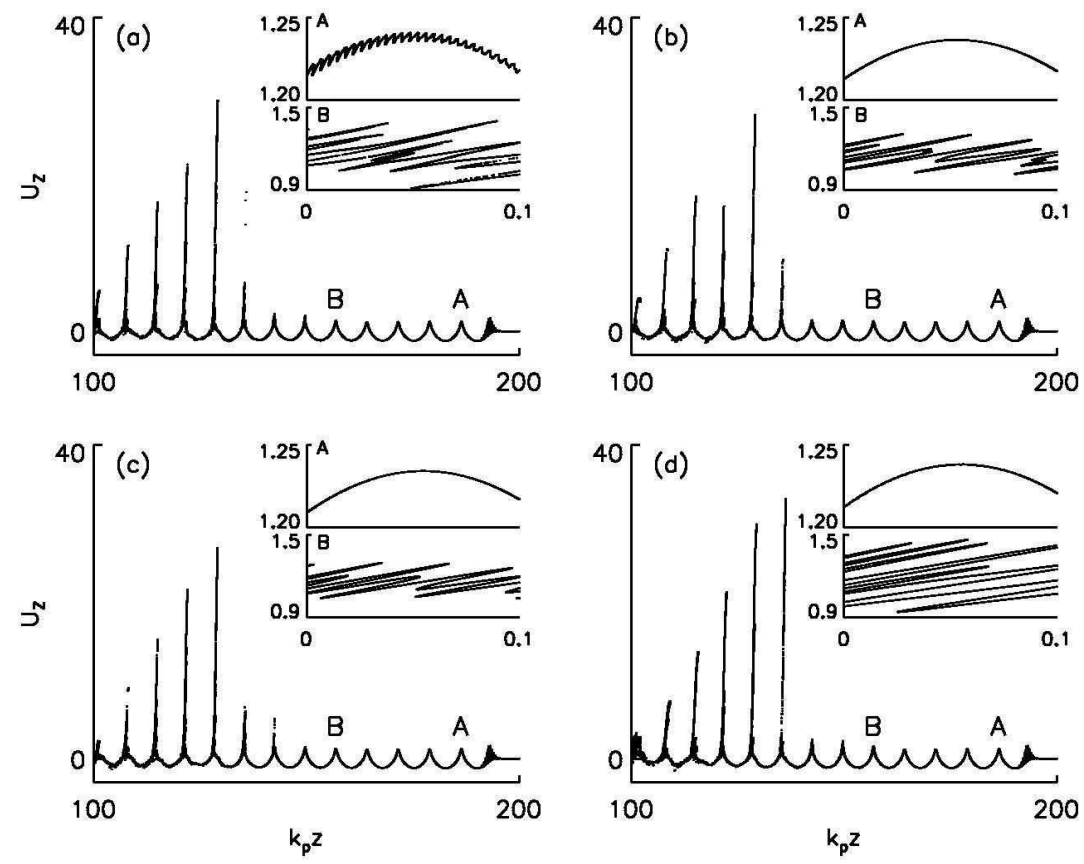

FIGURE 5. Effect of interpolation method and filtering on the macro-particle phase space of a laserdriven plasma wave in 1-D for an initially cold plasma. The longitudinal grid spacing used was $\lambda_{0} / 36$ with 400 macro-particles per cell and various interpolation methods: (a) linear interpolation; (b) quadratic interpolation; (c) cubic interpolation; and (d) linear interpolation with a $(1,2,1)$ filter plus compensator applied to the current. Insets show details of phase space at the first (A) and fifth (B) buckets after the laser pulse. See Fig. 8 of Ref. [12].

fidelity but not to more accurate (in the formal sense) solutions.

\section{CONCLUSIONS}

The cold fluid equations (assuming an initial delta-function momentum distribution), coupled to Maxwell equations, are an exact representation of the Vlasov-Maxwell equations for a cold initial condition. Hence, for a cold initial condition, the PIC algorithm should reproduce the cold fluid solution, and there should be no heating or trapping, provided no shocks have developed. This is true in any number of dimensions. Heating or trapping would violate entropy conservation, which is an exact constant of the Vlasov-Maxwell equations. The appearance of shocks would indicate that the collisionless assumption is no longer valid, since collisions are required for energy transport across shocks. Shock development indicates that the Vlasov model (the basis of the collisionless PIC algorithm) is no longer valid, and a collisional Boltzmann model of the plasma is required.

Based on the observed numerical heating in PIC for a cold initial condition, it seems plausible that the evolution of the plasma generated by the PIC algorithm depends critically on the initial condition. It has been shown that for thermal initial conditions, the PIC algorithm can converge to the correct plasma response [6]. For the cold initial condi- 
tion, we see evolution that is inconsistent with known properties of the Vlasov-Maxwell equations. This naturally leads to the suggestion that the solution spaces generated by the PIC algorithm for cold and warm initial conditions are disjoint, with only warm initial conditions leading to physically correct behavior. This conclusion is bolstered by the Lax Equivalence Theorem [11]: consistency and stability are both required to guarantee convergence. While the PIC algorithm appears to be formally consistent with the Vlasov-Maxwell equations, the phase-space structures that develop lead to trapping by triggering the linear grid heating instability. This numerical instability formally violates the conditions of the Lax theorem, leading one to believe that for cold initial conditions there is no justification in expecting the PIC algorithm to converge to a solution of the Vlasov-Maxwell equations. This is not a rigorous result, as such would require an existence proof for solutions of the relativistic Vlasov-Maxwell equations, nonetheless, it is troubling as it seem highly unlikely that the Lax theorem in some form would not apply.

\section{ACKNOWLEDGMENTS}

The authors would like to thank J-L. Vay, D. Bruhwiler, E. G. Evstatiev, E. CormierMichel, and E. Esarey for useful discussions. Work at UNL was supported by the Director, Office of Science, Office of Fusion Energy Sciences, of the U.S. Department of Energy under Contract No. DE-FG02-08ER55000. Work at LBNL was supported by the Director, Office of Science, Office of High Energy Physics, of the U.S. Department of Energy under Contract No. DE-AC02-05CH11231.

\section{REFERENCES}

1. C. K. Birdsall, A. B. Langdon, V. Vehedi, and J. P. Verboncoeur, Plasma Physics via Computer Simulations, Adam Hilger, Bristol, Eng., 1991.

2. F. H. Harlow, A machine calculation method for hydrodynamic problems, Tech. Rep. LAMS-1956, Los Alamos Scientific Laboratory (1955).

3. C. Birdsall, IEEE Trans. Plasma Sci. 19, 65-85 (1991).

4. J. P. Verboncoeur, Plasma Phys. Control. Fusion 47, A231-A260 (2005).

5. B. A. Shadwick, G. M. Tarkenton, and E. H. Esarey, Phys. Rev. Lett. 93, 175002 (2004).

6. B. A. Shadwick, G. M. Tarkenton, E. H. Esarey, and C. B. Schroeder, Phys. Plasmas 12, 056710 (2005).

7. C. B. Schroeder, E. Esarey, B. A. Shadwick, and W. P. Leemans, Phys. Plasmas 13, 033103 (2006).

8. E. Esarey, C. B. Schroeder, E. Cormier-Michel, B. A. Shadwick, C. G. R. Geddes, and W. P. Leemans, Phys. Plasmas 14, 056708 (2007).

9. R. W. Hockney, and J. W. Eastwood, Computer Simulation using Particles, Taylor \& Francis Group, New York, 1988.

10. A. B. Langdon, J. Comp. Phys. 6, 247-267 (1970).

11. R. Richtmyer, and K. W. Morton, Difference Methods for Initial Value Problems, Wiley-Interscience, 1967.

12. E. Cormier-Michel, B. A. Shadwick, C. G. R. Geddes, E. Esarey, C. B. Schroeder, and W. P. Leemans, Phys. Rev. E 78, 016404 (2008).

13. J. M. Dawson, Phys. Rev. 118, 381-389 (1960).

14. C. S. Gardner, Phys. Fluids 6, 839-840 (1963).

15. J. Villasenor, and O. Buneman, Comput. Phys. Commun. 69, 306 (1992).

16. T. Z. Esirkepov, Comput. Phys. Commun. 135, 144 (2001). 